\title{
Evaluación de la pertinencia del título universitario "Todos Somos Campus" dirigido a personas con discapacidad intelectual
}

\author{
Mạ Luisa BELMONTE \\ Lucia MIRETE \\ Begoña GALIÁN
}

Datos de contacto:

Ma Luisa Belmonte

Universidad de Murcia

marialuisa.belmonte@um.es

Lucia Mirete

Universidad de Alicante

Luicia.mirete@gmail.com

Begoña Galián

Universidad de Murcia

Begona.g.n@um.es

\section{RESUMEN}

El título universitario de Estudios Propios "Todos Somos Campus", cofinanciado por el Fondo Social Europeo y la Iniciativa Empleo Juvenil, a través de la convocatoria de ayudas de Fundación ONCE, permite que jóvenes con discapacidad intelectual se formen en la Universidad de Murcia y se preparen para conseguir un empleo.

El programa formativo se desarrolla entre octubre del 2019 y junio de 2020. A lo largo de ese periodo, los 17 jóvenes con discapacidad intelectual se incorporan a las aulas y la vida universitaria a través de la construcción de itinerarios formativos integrales, accesibles y personalizados.

El abordaje de contenidos se realiza de forma aplicada y transversal, e incluye un periodo de prácticas que permite al alumnado interactuar con empresas colaboradoras. La metodología de trabajo está basada en grupos de trabajo colaborativos, de apoyo entre iguales, con metas establecidas y completamente accesibles y flexibles, adaptándose a los requerimientos de los contenidos, y permitiendo el establecimiento de sinergias entre ellos, actividades en cadena, trabajo en gran grupo, entre otros.

Todos Somos Campus supone una formación para la mejora de la autonomía y la inclusión socio-laboral, sin olvidar que también desarrolla la motivación por el aprendizaje y desempeño responsable de tareas, pudiendo afirmar que la Universidad de Murcia apuesta por establecer un sistema de formación inclusivo y de normalización comunitaria.

PALABRAS CLAVE: discapacidad intelectual; universidad; inclusión; empleo. 


\title{
Evaluation of the university degree relevance "We Are All Campus" aimed for people with intellectual disabilities
}

\begin{abstract}
The university degree of Own Studies "We are All Campus", co-financed by the European Social Fund and the Youth Employment Initiative, through the call for grants from the ONCE Foundation, allows young people with intellectual disabilities to be trained at the University of Murcia and prepared to get a job.

The training program takes place between October 2019 and June 2020. Throughout this period, the 17 young people with intellectual disabilities are incorporated into classrooms and university life. They are integrated through the construction of comprehensive, accessible, and personalized training itineraries.

The content approach is carried out in an applied and cross-sectional way, which includes a period of practices allowing students to interact with collaborating companies. The working methodology is based on collaborative work groups, peer support, with fixed goals, fully accessible and flexible; being adapted to content requirements, and being able to establish synergies between participants, chain activities, large group work, among others.

We are all Campus means training to improve autonomy and socio-labor inclusion, bearing in mind that it develops motivation for learning and responsible task performance; in order to say that the University of Murcia aims to establish a system for the inclusive training and community standardization.
\end{abstract}

KEYWORDS: intellectual disability; university; inclusion; employment.

\section{Luces y sombras de la discapacidad intelectual}

Desde la creación de la Convención sobre los derechos de las personas con discapacidad, se viene acaeciendo un cambio de perspectiva hacia un modelo social, que aboga por entender la discapacidad como construcción social, y no como característica personal (Manzanera y Brändle, 2019). Durante décadas, la discapacidad luchó por encontrar un hueco en el mundo de la investigación educativa (Meekosha y Shuttleworth, 2009), dada la necesidad de construir nuevos pilares sociales (Rocco, 2011). El objetivo primordial fue redefinir conceptos erróneos entre jerarquías de discapacidades, así como la relación entre personas con y sin discapacidad, como términos descriptivos (Vehmas y Watson, 2014).

Históricamente, la educación universitaria y el colectivo de estudiantes con discapacidad intelectual, no han sido entes demasiado compatibles (Brewer y Movahedazarhouligh, 2019). En los últimos años, las instituciones de 
educación superior, de manera ascendente, han ofrecido oportunidades para que el alumnado con discapacidad intelectual participe en la vida del campus (Ryan, Randall, Walters y Morash-MacNeil, 2019). Esto ha supuesto un significativo avance en materia de inclusión educativa (Melero, Moriña y Perera, 2019), que se ha visto reflejado en el aumento de los estudios sobre la respuesta de la educación superior a esta nueva situación de inclusión de los jóvenes con discapacidad en la Universidad (Arnaiz, 2019; Claiborne, Cornforth, Gibson, y Smith, 2010; Fuller, Bradley y Hall, 2006; Fuller, Bradley y Healey, 2004; Fuller, Healey, Bradley y Hall, 2004; Holloway, 2001; Hong, Haefner, y Slekar, 2011; Hopkins, 2011; Moriña, y Perera, 2018; Moswela y Mukhopadhyay, 2011; Mullins y Preyde, 2013; Riddell, Tinklin y Wilson, 2005; Riddell, Wilson y Tinklin, 2002; Ryan y Struths, 2004; Shevlin, Kenny y McNeela, 2004; Taylor, Baskett y Wren, 2010).

La integración se fundamenta en la normalización de la vida del estudiante con necesidades educativas especiales (Barrio de la Puente, 2009), pero, una vez completada la educación secundaria, no es usual esperar que la persona con discapacidad prolongue su andadura por las sendas de la educación superior (Gillan y Coughlan, 2010; Grigal y Hart, 2010; Mock y Love, 2012). Por ello, todavía es largo el camino a recorrer (Castellana y Sala, 2006; Díaz y Funes, 2016; Muñoz, Novo y Espiñeira, 2013), sabiendo, además, que el abandono, o interrupción educativa (Van Dijk, 2012) de estos estudiantes es más frecuente (Sánchez-Gelabert y Elias, 2017).

Por otro lado, el empleo es un elemento crucial para fomentar el desarrollo de la calidad de vida de las personas con discapacidad intelectual (Ryan, Randall, Walters y Morash-MacNeil, 2019), pese a los retos, de muy distinta naturaleza, que esta aspiración conlleva (Echeita y Simón, 2014). La integración al mercado de trabajo es una de las claves principales para la normalización (Manzanera y Ortiz, 2017), junto con su integración en la sociedad (Manzanera y Brändle, 2019). Sin embargo, el acceso laboral no es equitativo (Sánchez, Hernández y Raya, 2016), siendo en general la situación de este colectivo, peor que la de las personas sin discapacidad (Manzanera y Ortiz, 2017; Ortiz y Olaz, 2018).

\section{El programa Todos Somos Campus (TSC)}

En el marco de la Estrategia Europa 2020, esbozada encauzando sus miras a la creación de unas condiciones propicias para una economía más competitiva y creadora de empleo, generando un crecimiento inteligente, sostenible e inclusivo, parece necesario contar con programas afines que refuercen esta Estrategia, planteando actuaciones encaminadas al empleo de los sectores de población más desfavorecidos.

Desde la Universidad de Murcia se plantea el Título Universitario de Estudios Propios Todos Somos Campus (TSC). Una iniciativa que quiere sumarse al 
esfuerzo social para favorecer y facilitar la incorporación del colectivo de jóvenes con discapacidad intelectual al mercado laboral. Con este fin, comienza a fraguarse un proyecto formativo para el empleo, capaz de dar respuesta a las necesidades individuales de este colectivo, construyendo itinerarios formativos integrales y personalizados para que los jóvenes con discapacidad intelectual puedan participar como sujetos de derecho en su comunidad, favoreciendo sus potencialidades y oportunidades laborales.

Los objetivos de este programa son ambiciosos:

- Proporcionar formación encaminada a la mejora de la autonomía y la preparación laboral.

- Facilitar recursos para el aumento de la inclusión laboral y social del colectivo.

- Dotar a los participantes de una formación integral, humanista y laboral polivalente maximizando sus posibilidades de acceso y mantenimiento en el mercado de trabajo.

- Establecer un sistema de formación inclusivo y de normalización en el marco de la comunidad universitaria.

- Desarrollar la motivación y el interés de los participantes por el aprendizaje y el desempeño de las distintas tareas, con eficacia y responsabilidad.

Por otro lado, los destinatarios del programa son 17 jóvenes de entre 18 a 30 años que deben cumplir con los requisitos de: estar inscritos en el Sistema de Garantía Juvenil; presentar discapacidad intelectual igual o superior al 33\% reconocida oficialmente por el Instituto Murciano de Acción Social (IMAS); poseer capacidad de desplazamiento autónomo; y tener habilidades académicas básicas de competencias mínimas en lectoescritura y cálculo.

Las competencias que el curso propone versan en torno a la adquisición de habilidades y actitudes que cualquier ciudadano, en edad laboral, deberia poseer, como son:

- Aplicar los conceptos, teorías y principios adquiridos en la resolución de problemas y toma de decisiones.

- Adquirir compromisos sociolaborales, que contribuyan a su crecimiento como ciudadanos de pleno derecho.

- Adquirir y manifestar habilidades sociales y emocionales necesarias para relacionarse con éxito en los diferentes entornos en los que se desenvuelvan y trabajar satisfactoriamente en equipo

- Adquirir habilidades de manejo de tecnologías de la información y la comunicación, que permitan resolver con eficacia tareas relacionadas con su trabajo.

- Desarrollar actitudes flexibles con capacidad de adaptación al cambio. 
Evaluación de la pertinencia del título universitario "Todos Somos Campus" dirigido a personas con discapacidad intelectual

Estructuración del curso en tres bloques: Desarrollo Personal y Autonomía, Formación para el Empleo y Desarrollo Profesional

Insertas dentro del primero de ellos, centrado en el Desarrollo Personal y la Autonomia, se encuentran cuatro grandes temáticas, básicas para una inclusión social plena:

i. Habilidades para la comunicación.

Imagen personal (cuidado e higiene personal, vestimenta, adecuación al lugar y al momento apropiado, buenos hábitos).

La comunicación verbal y no verbal (habilidades específicas de comunicación, habilidades conversacionales, espacio y contacto físico, expresión facial, postura corporal, tono-timbre-intensidad y prosodia de la voz, expresión de sentimientos).

Lenguaje y expresión comunicativa (reglas básicas del proceso de comunicación oral, elementos y funciones de la comunicación oral, sistema de comunicación alternativa, obstáculos en la comunicación, seguir y dar instrucciones, hacer peticiones, saber expresarse con asertividad.

La comunicación escrita y la lectura (usos y formas, la escritura como medio de comunicación, aspectos gramaticales elementales sobre ortografia y sintaxis, la disposición espacial de la escritura, funcionalidad del lenguaje escrito, lectura global de carteles, iconos, formatos establecidos calendarios, horarios, instancias, nombres y apellidos, etc), reglas de elaboración de textos en lectura fácil, lectura comprensiva: la palabra, la frase, el párrafo, etc., la lectura como su uso, entretenimiento y disfrute.

\section{ii. Habilidades sociales.}

Iniciativa y participación (habilidades sociales básicas, normas de cortesía, ritmo de trabajo, rendimiento, calidad, actitud cooperativa, actitud de ayuda y conocimiento del grupo).

Autocrítica y aceptación de propuestas de otros (identificación de puntos fuertes y a mejorar, pedir ayuda y/o aclaraciones, aceptar compromisos, tolerancia y respeto a los demás).

Resolución de problemas (tipos de problemas, escucha activa, análisis de las consecuencias, habilidades de negociación en los acuerdos, habilidades alternativas a la agresión).

Trabajo en equipo (tipos de equipos, tipos de liderazgo, principios para trabajar en equipo, la toma de decisiones compartida y el cumplimiento de tareas). 
Gestión del conflicto (detección y tipos de conflicto, situaciones que provocan conflicto, resolución de conflictos, la toma de decisiones, la empatía y la negociación).

\section{iii. Habilidades emocionales.}

Autoconocimiento y autoestima (emociones negativas y pensamientos erróneos, motivación para el trabajo, derechos y deberes de las personas con discapacidad intelectual, marco legal y toma de decisiones).

Empoderamiento personal (adaptación y satisfacción laboral, aceptación social, el desempeño sociolaboral y los tipos de actitudes).

Toma de decisiones (establecer objetivos, recoger información, valorar soluciones, planificación a corto, medio y largo plazo).

Control del estrés y ansiedad (higiene del sueño, técnicas de relajación, autoinstrucciones y autocontrol).

\section{iv. Entrenamiento cognitivo.}

Reflexión y autoaprendizaje (autocontrol, exigencia y reconocimiento del proceso de aprendizaje, la confianza y perseverancia, el afrontamiento de los resultados).

Atención y memoria (tipos de atención y de memoria, establecimiento de rutinas, la supervisión y el repaso, estrategias de mejora, orientación espacio temporal).

Habilidades de planificación (funciones ejecutivas, razonamiento, la gestión del tiempo, la división y organización del trabajo y la evaluación de resultados).

Gestión por procesos (perfiles profesiones, procesos en el puesto de trabajo, estándares e indicadores laborales, la coordinación en el trabajo y la mejora continua).

Dentro del segundo bloque, de Formación para el Empleo, se agrupan dos tópicos imprescindibles de cara a un futuro laboral:

\section{v. Habilidades laborales.}

Comunicación personal y laboral (normas de convivencia, tipos de relaciones, perfiles comunicativos, las relaciones en horario en el trabajo y en el descanso).

Organización y normativa (legislación laboral (derechos y deberes de los trabajadores, el contrato y tipos de contrato, seguridad social, etc., jornada y 
salario, seguridad social), puntualidad, asiduidad, responsabilidad, permisos, horarios, etc., conocimiento y uso del teléfono, correos e internet).

Prevención de riesgos (higiene postural, salud laboral, protección individual, uso responsable de equipos y normas básicas de seguridad).

Orientación al cliente (la atención personal, el cliente satisfecho, cómo ofrecer información y cómo ser prosocial).

Ética profesional (discreción profesional, profesionalidad, eficacia, eficiencia, dignidad, solidaridad y transparencia).

\section{vi. Habilidades especificas.}

Matemáticas (numeración (números naturales y enteros, negativos, decimales, utilidad de los números: contar, aproximar, ordenar, aplicación y resolución de problemas de la vida cotidiana).

Cálculo (adición, sustracción, multiplicación y división de números naturales, uso de la calculadora, cálculo mental, aplicación y resolución de problemas de la vida cotidiana), medir (unidades de medida, aplicación y resolución de problemas de la vida cotidiana), pesar (unidades de peso, aplicación y resolución de problemas de la vida cotidiana), el sistema monetario (el dinero, transacciones económicas responsables en contextos reales), orientación temporal (las medidas del tiempo: siglo, año, meses, días, horas, minutos, aplicación y resolución de problemas de la vida cotidiana).

Tecnología (conceptos y terminología de la informática (hardware, software, CPU, dispositivos de entrada, dispositivos de salida, principales dispositivos de entrada y salida, introducción a los elementos del escritorio, iconos, botones de inicio, etc., digitalización de documentos), introducción al paquete Office (Word, Excell, Access, Power Point), Internet (los buscadores, correo electrónico, páginas web, redes sociales, uso responsable).

El último bloque de contenidos, que abarca el Desarrollo Profesional, congrega temáticas fundamentales y concretas de formación especializada orientada al empleo:

vii. Orientación vocacional laboral.

Preferencias profesionales (proyecto personal y profesional-toma de decisiones) y la formación para el empleo (oferta formativa de la Región de Murcia).

Búsqueda de información para encontrar un puesto de trabajo, técnicas e instrumentos para la búsqueda de empleo (el currículum, carta de presentación, pruebas psicotécnicas, entrevista, cumplimentar instancias), aproximación a los diferentes sectores profesionales, inserción laboral). 
viii. Desarrollo profesional específico.

Operaciones Auxiliares de Servicios: tramitación, de correspondencia y paquetería, control de almacén de material de oficina y reprografia, operaciones básicas de tesorería, comunicación telefónica en el ámbito profesional, recepción de personas externas a la organización, archivo de documentos, reprografia de documentos, integración y comunicación en el centro de trabajo.

Aproximación a las diferentes ocupaciones y puestos de trabajo ofertados en el prácticum.

\section{ix. Prácticum (empleo con apoyo):}

Se les proporcionarán experiencias de prácticas formativas, utilizando la metodología de empleo con apoyo, dentro del ámbito universitario y/o empresarial en las siguientes ocupaciones: Auxiliar de servicios generales; Auxiliar de archivo; Ordenanza; Auxiliar de información; Telefonista en servicios centrales de información; Clasificador y/o repartidor de correspondencia; Auxiliar de venta; Empleado de reposición; Operario de reprografia; Operario de acabados de reprografia; Preparador de pedidos.

\section{x. Formación individualizada orientada al empleo}

Autoconocimiento y conocimiento del mercado de trabajo (¿qué competencias tengo y qué necesitan las empresas?, concepto de empleabilidad, sectores económicos y empresas en las que buscar empleo, fuentes de información sobre el mercado de trabajo).

Búsqueda de empleo: planificación inicial para la búsqueda de empleo: recursos útiles y definición de objetivos y elaboración de un plan de acción.

Herramientas para la búsqueda de empleo: curriculum vitae: elaboración de $\mathrm{CV}$, elaboración de video curriculum, curriculum Europass.

Entrevista de trabajo. las claves para afrontar una entrevista de trabajo, simulación de entrevistas de trabajo con los alumnos.

Gestión de la red de contactos: elaboración de nuestra autopresentación, estrategias para ampliar y aprovechar nuestra red de contactos.

\section{La metodología docente}

La metodología de aula habitual, en el programa, está basada en grupos de trabajo colaborativos. En ella, los participantes son distribuidos en pequeños grupos de tres o cuatro componentes, en función de las características personales detectadas en la evaluación psicopedagógica inicial. 
Evaluación de la pertinencia del título universitario "Todos Somos Campus" dirigido a personas con discapacidad intelectual

El funcionamiento de estos grupos permite una metodología activa, de apoyo entre iguales, con metas establecidas para cada grupo y completamente colaborativa y solidaria. El abordaje de cada bloque de contenidos se diseña de forma aplicada y transversal, dirigido a la resolución de problemas concretos.

La combinación de estos grupos colaborativos se plantea de forma flexible, adaptándose a los requerimientos de los contenidos, pudiendo establecerse sinergias entre ellos, actividades en cadena, trabajo en gran grupo, etc., en función del mejor aprovechamiento docente. Esta dinámica permite el abordaje combinado de bloques de conocimientos, estableciendo un sistema docente transversal vasto y ajustado a la realidad. De este modo, se desarrolla la creatividad y la capacidad de autodeterminación, propiciando de forma sistemática espacios y momentos para que el alumnado exprese sus opiniones e intereses de forma democrática.

Se plantea el uso de dispositivos informáticos de última generación (tabletas, apps, smartphones) integrados en la actividad del aula a diario, con recursos digitales generados ad hoc, garantizando su accesibilidad a medida del colectivo.

Incluidos en la programación de contenidos, se consideran también, seminarios específicos, donde se dispone de la presencia de expertos en temas tratados con el fin de aportar un punto de vista directo de implicados en el mismo, para así, profundizar en el tema. Dentro de estos seminarios se incluyen casos de éxito, pudiendo mostrar, de esa manera, a los participantes, aquellos jóvenes con discapacidad intelectual incorporados al mercado laboral que narran en primera persona, sus experiencias, problemas, etc.

Para la docencia, se considera necesario contar, además de un profesorado especializado en los contenidos, con la presencia simultánea de personal técnico de apoyo experto en discapacidad intelectual. La combinación de ambos en el aula, permite mantener un ritmo docente adecuado y garantizar que todos los grupos mantengan el interés en los contenidos.

Se diseña un sistema de tutoría y guiado triple, formado por:

- Tutor de contenidos: profesor responsable de cada asignatura, estableciendo un sistema de tutorias individualizadas de asistencia obligatoria para los participantes, destinando una sesión semanal para ello. Además de esta jornada, los participantes dispondrán de acceso a los recursos de la UMU para la realización de tutorías virtuales con el tutor de contenidos.

- Tutor personal: especialista en discapacidad intelectual, asignado de forma individualizada a partir de la evaluación psicopedagógica inicial, y que acompañará al estudiante a lo largo del programa, 
proporcionando apoyo tanto al alumno/a como a su familia con reuniones regulares.

- Tutor de refuerzo: estudiante universitario voluntario, asignado de forma individualizada, tras convocatoria abierta, con especial énfasis para los centros objetivo (Psicología, Educación Social, Trabajo Social y Relaciones Laborales), encargado de apoyar de forma semanal el abordaje de los contenidos y el trabajo personal del alumno, motivándolo para la consecución de las metas marcadas.

Tras el cierre de cada tema de contenidos, se realiza una reunión entre los tres tutores, analizando la evolución de cada participante de forma individualizada, atendiendo a su esfuerzo, motivación y adquisición de contenidos.

Por otra parte, los alumnos participantes deben preparar una intervención individualizada a realizar en los estudios ya citados (Psicología, Educación, Educación Social, Trabajo Social y Relaciones Laborales) en asignaturas afines a la inclusión educativa y laboral con el fin de compartir su experiencia educativa, expectativas laborales, metas, etc., con los estudiantes universitarios.

\section{Recursos profesionales, espaciales y otras colaboraciones}

Con respecto a las instalaciones, cabe destacar que la actividad docente se realiza en los aularios e instalaciones vinculadas a los centros de la UMU, participantes en el programa, como son las Facultades de: Psicología, Educación, Educación Social, Trabajo Social y Relaciones Laborales. Dichos espacios están equipados con recursos más que suficientes para el desarrollo de las metodologias docentes previstas.

El personal docente es profesorado universitario que, mediante oferta de participación abierta en la Universidad de Murcia, ha manifestado su interés en participar $y$, posteriormente, ha sido seleccionado con base en su experiencia relacionada con los contenidos docentes del título propio TSC, así como en atención a la diversidad.

Con antelación a la actividad docente, todo el profesorado seleccionado recibe formación especializada sobre el colectivo de jóvenes con discapacidad, sus características y habilidades, así como recursos a tener en cuenta para el trabajo en el aula con ellos.

Por otro lado, el curso está planificado para contar con dos perfiles de equipo técnico: 
Evaluación de la pertinencia del título universitario "Todos Somos Campus" dirigido a personas con discapacidad intelectual

- Personal de apoyo en el aula: profesionales especializados en el trabajo y la formación a jóvenes con discapacidad intelectual, mediante convocatoria abierta a interesados. Para su selección se tiene en cuenta la vinculación a asociaciones u organizaciones del ámbito de la discapacidad intelectual, y su experiencia en formación para el empleo en discapacidad intelectual.

- Personal técnico especialista UMU: por un lado, Técnicos de Atención a la Diversidad y Voluntariado (ADyV), encargados de la coordinación de actuaciones del programa, su plan de estudios, y el seguimiento y supervisión del primer bloque de contenidos del programa con sus dinámicas docentes, garantizando así la adecuación de su desarrollo a los objetivos del mismo. Por otro lado, Técnicos de Orientación Laboral, pertenecientes al Centro de Orientación e Información de Empleo (COIE), encargados de la planificación, desarrollo y evaluación del periodo de prácticas, así como el seguimiento y supervisión del segundo y tercer bloque de contenidos del programa con sus dinámicas docentes, garantizando así mismo, la adecuación de los objetivos al mismo.

Los Programas de Apoyo transversales son cuatro:

Programa Tutor: tutoría individualizada realizada por estudiantes universitarios voluntarios, preferiblemente, con algún tipo de discapacidad que no afecte al proceso de la tutoría.

Programa Apuntes: apoyo para la realización de fotocopias, apuntes de clase, encuadernaciones, ampliaciones y materiales específicos.

Programa de Ayudas Técnicas: préstamo de equipos informáticos adaptados (en este caso tabletas para cada grupo docente).

Programa de Apoyo Voluntario: programa de inclusión en la vida universitaria mediante voluntarios de apoyo, favoreciendo la participación de los alumnos en actividades deportivas, culturales y sociales de la UMU.

Por último, se destaca la colaboración de la comunidad universitaria, dado que se organiza desde el Vicerrectorado de Formación e Innovación, el Vicerrectorado de Estudiantes y el Vicerrectorado de Transferencia, Emprendimiento y Empleo, por medio del Servicio de Atención a la Diversidad y Voluntariado y el Centro de Orientación e Información de Empleo.

Las entidades sociales relacionadas con la discapacidad intelectual que colaboran son: Comité de Representantes de Personas con Discapacidad y sus Familias de la Región de Murcia; Federación Plena Inclusión; Asociación CEOM (Asociación para la Integración de Personas con Discapacidad Intelectual), y la Fundación Síndrome de Down de la Región de Murcia. 
La gestión y organización del Practicum en empresas e instituciones se lleva a cabo por el COIE de la Universidad de Murcia, el cual tiene, entre otras responsabilidades, la promoción y gestión de las prácticas extracurriculares de los estudiantes de las titulaciones oficiales y propias de la Universidad de Murcia. Gestiona más de 2.700 prácticas externas cada curso académico y posee Convenios de Cooperación Educativa con más de 9.300 empresas e instituciones.

Para la realización de prácticas externas, la Universidad de Murcia y las empresas e instituciones cuentan con un Convenio de Cooperación Educativa, como requisito previo e imprescindible para la incorporación de los estudiantes en prácticas. A través de este Convenio de Cooperación Educativa se establece el marco regulador de las relaciones entre el estudiante, las empresas/instituciones y la Universidad de Murcia.

\section{Consideraciones finales}

E1 concepto de educación inclusiva puede ser definido de varias formas (Ainscow, Farrell y Tweddle, 2000; Dyson, 2001; Echeita, 2008, Escudero y Martínez, 2011) aunque, de un modo u otro, todas ellas convergen en vislumbrar la diversidad como un reto educativamente enriquecedor (García Hernández, 2004), que repercute directamente en la comunidad y favorece el cambio social (Laluvein, 2010; Booth y Ainscow, 2000; Echeita, 2008).

A nivel empresarial, cada vez con mayor frecuencia se demanda que estos actores sociales sean ejemplo de valores morales y éticos (Tejedor, 2015). La inserción laboral de la persona con discapacidad se logra, en gran medida, mediante el empleo protegido, y dentro del empleo protegido, los centros especiales de empleo son el instrumento más importante. En ellos la estabilidad laboral es superior a la de la empresa ordinaria (Barea y Monzón, 2008). Estos centros se configuran así, como la mayor oportunidad de empleo que encuentran respecto de la inserción laboral (Rodríguez, García y Toharia, 2009).

Las personas con discapacidad, al incorporarse al mercado laboral, experimentan diversos cambios a nivel emocional, asi como mejoría madurativa y autonomía personal, contribuyendo todo ello a configurar la identidad adulta de estas personas, al dotarlas de autosuficiencia (Pallisera y Rius Bonjoch, 2007).

El Título Universitario de Estudios Propios TSC, cofinanciado por el Fondo Social Europeo y la Iniciativa Empleo Juvenil a través de la convocatoria de ayudas de Fundación Organización Nacional de Ciegos Españoles (ONCE), permite que jóvenes con discapacidad intelectual se formen en la Universidad de Murcia y se preparen para conseguir un futuro laboral. Este programa supone una formación para la mejora de la autonomía e inclusión socio- 
Evaluación de la pertinencia del título universitario "Todos Somos Campus" dirigido a personas con discapacidad intelectual

laboral, así como también desarrolla la motivación por el aprendizaje y desempeño responsable de tareas.

Para la Universidad de Murcia supone establecer un sistema de formación inclusivo y de normalización en el entorno de la comunidad universitaria con los correspondientes beneficios que esto conlleva, tales como sociales, afectivos y de equilibrio personal, relativos al sentimiento de no exclusión (Borland y James, 1999); académicos o de educación para la justicia social (Riddell, Tinklin y Wilson, 2005); y, por último, laborales o de empleabilidad, concernientes al número, tipo y permanencia en el empleo logrado por la persona con discapacidad (Ryan y Struths, 2004).

Algunos sectores universitarios tienden a alejar a los estudiantes con discapacidad debido a la falta de conocimiento, capacitación y conciencia de la misma (Bunbury, 2018). Ahora bien, diferentes estudios han confirmado que este tipo de programas implicarán, en el futuro, una mayor tasa de empleo y vida independiente para los estudiantes con discapacidad (Ryan, Randall, Walters y Morash-MacNeil, 2019). En algunos casos la inserción laboral puede incluso conllevar la emancipación de la familia, por parte de estas personas (Pallisera y Rius Bonjoch, 2007).

A modo de conclusión, se quiere señalar que, independientemente de las barreras y los obstáculos actitudinales y estructurales que pueden encontrar en múltiples ocasiones, las personas con discapacidad comienzan a ser bienvenidas en las instituciones de educación superior, llegando a formar parte de comunidad universitaria (Björnsdóttir, 2017), tal y como está ocurriendo en la Universidad de Murcia. Por todo ello, cabe concluir que la educación superior se presenta como un escenario más que adecuado, donde este sector estudiantil empieza a ser socialmente incluido, permitiéndole, incluso, reinventar una identidad que se haya podido ver deteriorada durante etapas previas en su recorrido educativo (Moriña, 2017).

\section{Referencias bibliográficas}

Ainscow, M., Farrell, P. y Tweddle, D. (2000): Developing policies for inclusive education: a study of the role of local education authorities. International Journal of Inclusive Education, 4(3), 211-229.

Arnaiz, P. (2019). La educación inclusiva en el siglo XXI. Avances y desafios. España: Universidad de Murcia.

Barea, J. y Monzón, J. (2008). Economía social e inserción laboral de las personas con discapacidad en el País Vasco. Bilbao: Fundación BBVA.

Barrio de la Puente, J. L. (2009). Hacia una educación inclusiva para todos. Revista complutense de educación, 20(1), 13-31. 
Björnsdóttir, K. (2017). Belonging to higher education: inclusive education for students with intellectual disabilities. European Journal of Special Needs Education, 32(1), 125-136. doi: 10.1080/08856257.2016.1254968

Booth, T. y Ainscow, M. (Eds.). (2000). Index for inclusion. Brighton, UK: Centre for Studies on Inclusive Education.

Borland, J. y James, S. (1999). The learning experience of students with disabilities in higher education: a case study of a UK university. Disability y Society, 14, 85-101.

Brewer, R. y Movahedazarhouligh, S. (2019). Students with intellectual and developmental disabilities in inclusive higher education: perceptions of stakeholders in a first-year experience. International Journal of Inclusive Education, 1-17.

Bunbury, S. (2018). Disability in higher education-do reasonable adjustments contribute to an inclusive curriculum?. International Journal of Inclusive Education, 1-16. doi: 10.1080/13603116.2018.1503347

Castellana, M. y Sala, I. (2006). Estudiantes con discapacidad en la universidad. Barcelona: Fundació Blanquerna Assistencial i de Serveis.

Claiborne, L., Cornforth, S., Gibson, A. y Smith, A. (2010). Supporting students with impairments in higher education: Social inclusion or cold comfort? International Journal of Inclusive Education, 15, 513-527. doi:10.1080/13603110903131747

Díaz, V. y Funes, S. (2016). Universidad inclusiva: reflexiones a partir de las experiencias de estudiantes con discapacidad de una universidad pública madrileña. Prisma Social, 16, 450-494.

Dyson, A. (2001). Dilemas, contradicciones y variedades de la inclusión. En M. Verdugo y F. Jordán de Urries (Eds.). Apoyos, autodeterminación y calidad de vida (pp. 145-160). Salamanca: Amarú.

Echeita, G. (2008). Inclusión y exclusión educativa. "Voz y quebranto". Revista Electrónica Iberoamericana sobre Calidad, Eficacia y Cambio en Educación, 6(2), 9-18.

Echeita, G. y Simón, C. (2007). La contribución de la educación escolar a la calidad de vida de las personas con discapacidad. Ante el desafio de su inclusión social. En R. de Lorenzo y L. Cayo Pérez Bueno. Tratado sobre Discapacidad (pp. 1103-1134) Madrid: Thomson y Aranzadi.

Escudero, J. M. y Martínez, B. (2011). Educación inclusiva y cambio escolar. Revista Iberoamericana de Educación, 55, 85-105.

Fuller, M., Bradley, A. y Hall, T. (2006). Listening to students: the experiences of disabled students of learning at university. In M. Adams y S. Brown 
Evaluación de la pertinencia del título universitario "Todos Somos Campus" dirigido a personas con discapacidad intelectual (Eds.), Towards Inclusive Learning in Higher Education: Developing Curricula for Disabled Students. London: RoutledgeFalmer.

Fuller, M., Bradley, A. y Healey, M. (2004). Incorporating disabled students within an inclusive higher education environment. Disability $y$ Society, 19, 455-468.

Fuller, M., Healey, A., Bradley, A. y Hall, T. (2004). Barriers to learning: a systematic study of the experience of disabled students in one university. Studies in Higher Education, 29(3), 303-318.

García Hernández, F. (2004). Una educación de calidad para los niños bien dotados desde la atención a la diversidad. De la educación integradora a la Educación Inclusiva. Revista Complutense de Educación, 15(2), 597619.

Gillan, D. y Coughlan, B. (2010). Transition from special education into postschool services for young adults with intellectual disability: Irish parents' experience. Journal of Policy and Practice in Intellectual Disabilities, 7(3), 196-203.

Grigal, M. y Hart, D. (2010). Think college! Postsecondary education options for students with intellectual disabilities. Baltimore, MD: Paul H. Brookes Publishing Company.

Holloway, S. (2001). The experience of higher education from the perspective of disabled students. Disability y Society, 16, 597-615.

Hong, B. S. S., Haefner, L. y Slekar, T. (2011). Faculty attitudes and knowledge toward promoting self-determination and self-directed learning for college students with and without disabilities. International Journal of Teaching and Learning in Higher Education, 23, 175-185.

Hopkins, L. (2011). The path of least resistance: A voice-relational analysis of disabled students' experiences of discrimination in English universities. International Journal of Inclusive Education, 15,711-727. doi: $10.1080 / 13603110903317684$

Laluvein, J. (2010). School inclusion and "community of practice". International Journal of Inclusive Education, 14(1), 35-48.

Manzanera, S. y Brändle, G. (2019). The Type of Disability as a Differential Factor in Entrepreneurship. Journal of Entrepreneurship Education, 22(S2), 1-10.

Manzanera, S. y Ortiz, P. (2017). Disability and its relationship with the labor market. Socio-labor situation. In: P. Ortiz y A. Olaz (Eds.). Entrepreneurship, employment and disability. A diagnosis (pp. 4585). Madrid: Aranzadi.

Meekosha, H. y Shuttleworth, R. (2009). What's so 'critical' about critical disability studies? Australian Journal of Human Rights, 15(1), 47-76. 
Melero, N., Moriña, A. y Perera, V. H. (2019). Acciones del profesorado para una práctica inclusiva en la universidad. Revista Brasileira de Educação, 24, 1-19. doi: 10.1590/s1413-24782019240016

Mock, M. y Love, K., (2012). One state's initiative to increase access to higher education for people with intellectual disabilities. Journal of Policy and Practice in Intellectual Disabilities, 9(4), 289-297.

Moriña, A. y Perera, V. H. (2018). Inclusive higher education in Spain: students with disabilities speak out. Journal of Hispanic Higher Education. doi: 10.1177/1538192718777360.

Moriña, A. (2017). "We aren't heroes, we're survivors": Higher education as an opportunity for students with disabilities to reinvent an identity. Journal of Futher and Higher Education, 41(2), 215-226. doi:10.1080/0309877X.2015.1070402

Moswela, E. y Mukhopadhyay, S. (2011). Asking for too much? The voices of students with disabilities in Botswana. Disability y Society, 26(3), 307319. doi:10.1080/09687599.2011.560414

Mullins, L. y Preyde, M. (2013). The lived experience of students with an invisible disability at a Canadian university. Disability $y$ Society, 28, 147-160. doi:10.1080/09687599.2012.752127

Muñoz, J. M., Novo, I. y Espiñeira, E. M. (2013). La inclusión de los estudiantes universitarios con discapacidad en las universidades presenciales: actitudes e intención de apoyo por parte de sus compañeros. Estudios sobre Educación, 24, 103-124.

Ortiz, P., y Olaz, A. (2018). Differential aspects in the entrepreneurship of people with disabilities. In A. Olaz y P. Ortiz (Eds.) Disability and entrepreneurship. Dimensions and interpretative contexts in qualitative key (pp. 39-54). Cizur Menor: Aranzadi.

Pallisera, M. y Rius Bonjoch, M. (2007). ¿Y después del trabajo, qué? Más allá de la integración laboral de las personas con discapacidad. Revista de Educación, 342, 329-348.

Riddell S., Tinklin T. y Wilson A. (2005). New Labour, Social Justice and Disabled Students in Higher Education. British Educational Research Journal, 31(5), 623-643.

Riddell, S., Wilson, A. y Tinklin, T. (2002). Disability and the wider access agenda: supporting disabled students in different institutional contexts. Widening Participation and Lifelong Learning, 4(3), 12-26.

Rocco, T. S. (2011). Challenging ableism, understanding disability, including adults with disabilities in workplaces and learning spaces. New Directions for Adult and Continuing Education (pp: 3-12). San Francisco: John Wiley y Sons. 
Evaluación de la pertinencia del título universitario "Todos Somos Campus" dirigido a personas con discapacidad intelectual

Rodriguez, G., Garcia, C. y Toharia, L. (2009). Evaluación de las politicas de empleo para personas con discapacidad y formulación y coste económico de nuevas propuestas de integración laboral. Madrid: Cinca.

Ryan, J. y Struths, J. (2004). University Education for All? Barriers to Full Inclusion of Students with Disabilities in Australian Universities. International Journal of Inclusive Education, 8, 73-90.

Ryan, J. B., Randall, K. N., Walters, E. y Morash-MacNeil, V. (2019). Employment and independent living outcomes of a mixed model postsecondary education program for young adults with intellectual disabilities, Journal of Vocational Rehabilitation, 50(1), 61-72. doi: 10.3233/JVR-180988

Sánchez, A., Hernández, M. y Raya, E. (2016). Inserción sociolaboral de las personas con discapacidad y modelos familiares. En D. Carbonero, E. Raya, N. Caparros y C. Gimeno (Coords). Respuestas transdisciplinares en una sociedad global. Aportaciones desde el Trabajo Social. Logroño: Universidad de La Rioja.

Sánchez-Gelabert, A. y Elias, M. (2017). Los estudiantes universitarios no tradicionales y el abandono de los estudios. Estudios sobre Educación, 32, 27-48. doi: 10.15581/004.32.27-48

Shevlin, M., Kenny, M. y Mcneela, E. (2004). Participation in higher education for students with disabilities: An Irish perspective. Disability y Society, 19(1), 15-30. doi:10.1080/0968759032000155604

Taylor, M., Baskett, M. y Wren, C. (2010). Managing the transition to university for disabled students. Education y Training, 52(2), 165-175.

Tejedor, J. (2015). La responsabilidad social corporativa y su aporte a la economía. EconLit, 11-25.

Van Dijk, S. (2012). La política pública para abatir el abandono escolar y las voces de los niños, sus tutores y sus maestros. Revista mexicana de investigación educativa, 17(52), 115-139.

Vehmas, S. y Watson, N. (2014). Moral wrongs, disadvantages, and disability: a critique of critical disability studies. Disability and Society, 29(4), 638650 . 